\title{
Cell cycle and related protein ${ }^{1}$
}

\author{
John Farley ${ }^{\mathrm{a}}$, Laurent Ozbun ${ }^{\mathrm{b}}$, Goli Samimi ${ }^{\mathrm{c}}$ and Michael J. Birrer ${ }^{\mathrm{b}, *}$ \\ a Department of Obstetrics and Gynecology, Uniformed Services University of the Health Sciences 4301 Jones \\ Bridge Road, Bethesda, MD 20814, USA \\ ${ }^{\mathrm{b}}$ Cell and Cancer Biology Department, Medicine Branch, Center for Cancer Research, National Cancer Institute, \\ Bethesda, MD 20892, USA \\ ${ }^{\mathrm{c}}$ Cancer Prevention Fellowship Program and Cell and Cancer Biology Branch, Center for Cancer Research, \\ National Cancer Institute, Bethesda, MD 20892, USA
}

Keywords: Gynecologic, malignancies, biologiomarkers

\section{Introduction}

Ovarian cancer is the most lethal gynecologic malignancy [1]. Despite the advent of multiple screening technologies, the majority of ovarian cancer patients still present at advanced stage, and the survival for patients with advanced stage disease is poor [2]. The recent genomic revolution has provided enormous information concerning the molecular characteristics of cancer. Identification and characterization of the genes and their protein products, which contribute to the malignant phenotype, can provide researchers with novel molecular targets which can be exploited in an attempt to improve ovarian cancer survival. In gynecologic oncology, we are now beginning to investigate these new biologic agents in the treatment of ovarian cancer [3]. There are a variety of cell surface receptors, signaling pathways, and nuclear proteins that stimulate cellular proliferation. An understanding of these biomarkers that affect growth deregulation in ovarian cancers can provide a framework for the rational application and

\footnotetext{
${ }^{1}$ The views expressed herein are those of the authors and do not reflect the official policy or opinion of the Department of Defense or the United States Army or Navy.

${ }^{*}$ Corresponding author: Michael J. Birrer, M.D., Ph.D., National Cancer Institute, Center for Cancer Research, Cell and Cancer Biology Department, 37 Convent Drive, Bldg 37 Room 1130, Bethesda, MD 20892, USA. Tel.: +1 301402 9586; Fax: +1 301480 4756; E-mail: birrerm@bprb.nci.nih.gov.
}

testing of these novel therapies [4]. This review will summarize the cell cycle biomarkers that affect growth deregulation in ovarian cancer and any association with prognosis and survival these proteins have been found to hold.

\section{Tumor suppressor genes}

Tumor suppressor genes produce protein products which function normally to inhibit a variety of aspects of neoplastic transformation to include cellular proliferation. These genes are usually inactivated through mutations which cause a loss of function and consequently a lack of inhibition of the respective cellular processes [5]. A variety of tumor suppressor genes have been evaluated in ovarian cancer. A summary of the prognostic significance of some of these tumor suppressor genes in ovarian cancer follows.

\section{1. $p 53$}

p53 is a tumor suppressor gene whose mutation is the most frequent genetic event described to date in ovarian cancer p53 inhibits cell cycle progression, particularly in respond to DNA damage [6,7]. Overexpression of p53 protein approaches $45 \%-55 \%$ of epithelial ovarian cancers [7-10]. p53 overexpression has been associated with advanced stage, higher grade, serous histology, and patient age $>61$ years. p53 protein expres- 
sion on multivariate analysis was an independent poor prognostic predictor of survival [9]. In other studies p53 protein overexpression in the univariate analysis was a significant poor prognostic factor; however after adjustment for stage, p53 overexpression did not retain statistical significance [7].

The Gynecologic Oncology Group sought to explain the apparent disparities in the literature regarding p53 protein overexpression and prognostic significance in epithelial ovarian cancer [11]. Overexpression $(>$ $30 \%$ ) of p53 protein occurred in $56 \%$ of tumors including $100 \%$ of patients with only missense mutation(s), $32 \%$ with truncation mutations, and $40 \%$ lacking a mutation in exons 2 to 11 . Overexpression of p53 was associated with tumor grade but not with patient outcome. Median survival for low p53 protein expression was 45 months while overexpression was associated with a median survival of only 39 months (Fig. 1) [11]. These results intimate that it is the mutation in the p53 gene, not overexpression of $\mathrm{p} 53$, that is the significant molecular genetic event, and can be associated with a short-term survival benefit [11].

\subsection{BRCA1}

BRCA1 is a putative tumor suppressor gene responsible for a hereditary breast ovarian cancer syndrome. While only responsible for approximately 5-10\% of all new ovarian cancer cases, mutation of breast and ovarian cancer susceptibility gene, BRCA-1, has been associated with a 40-63\% risk of developing ovarian cancer over a lifetime [12]. Normal ovarian surface epithelial cells express BRCA1 protein. However, decreased expression of BRCA1 was found in $16 \%$ of benign tumors, $38 \%$ of borderline tumors, and $72 \%$ of carcinomas [13]. Methylation of BRCAl was not detected in benign or borderline tumors, but was present in $31 \%$ of carcinomas. Reduced expression of BRCA1 protein correlated with the presence of gene methylation. Unfortunately, the prognosis of ovarian carcinoma patients did not correlate with BRCA1 protein expression or genetic status [13].

$\mathrm{LOH}$ at $17 \mathrm{q} 21$, the BRCA1 locus, is seen in $40-70 \%$ of invasive ovarian cancers [14]. Because of this, the frequency of BRCA1 promoter hypermethylation as an epigenetic means of BRCA1 inactivation has been evaluated for a large, population-based cohort of ovarian cancer patients [14]. BRCA1 hypermethylation was seen in only $15 \%$ of the sporadic cancers analyzed in this study. Interestingly, the BRCA1 methylation was only seen in ovarian cancer patients without a family history suggestive of a breast/ ovarian cancer syndrome. None of the 12 tumors with BRCA1 promoter hypermethylation demonstrated BRCA1 protein expression by immunohistochemistry. These findings suggested that reduced expression of BRCA1 protein along with genetic and epigenetic changes of the BRCA1 gene play an important role in the development of sporadic ovarian carcinomas. As a result, promoter hypermethylation may be an alternative to mutation in causing the inactivation of the BRCA1 tumor suppressor gene in sporadic ovarian cancer [14].

\section{3. $R b 2 / p 130$}

Retinoblastoma, $\mathrm{Rb}$, family members function as cell cycle regulators and modulate the sensitivity of cancer cells to chemotherapy [15-17]. Rb protein reactivity is found in approximately $47 \%$ of epithelial ovarian cancers [16]. Immunohistochemical analysis of 69 ovarian carcinomas for $\mathrm{Rb}$ protein expression revealed no association between $\mathrm{Rb}$ protein levels and relevant clinicopatholigic factors such as FIGO stage, grade, and histologic type [16]. pRb2/p130, however, is a member of the retinoblastoma gene family, and maps to human chromosome $16 \mathrm{q} 12.2$, a region in which deletions have been found in ovarian carcinoma [15]. Primary ovarian adenocarcinomas showed loss or decrease of $\mathrm{pRb} 2 / \mathrm{p} 130$ expression in $40 \%$ of tumors analyzed. $\mathrm{pRb} 2 / \mathrm{p} 130$ protein expression was inversely related to tumor grade with $73 \%$ of grade 1 and 2 cancers showing positive expression of $\mathrm{pRb} 2 / \mathrm{p} 130$ protein while $61 \%$ of grade 3 tumors were negative for protein expression [15]. This suggests that $\mathrm{pRb} 2 / \mathrm{p} 130$ may play a putative role as a tumor suppressor gene in ovarian cancer.

\section{4. $N m 23$}

Nucleoside diphosphate kinase/nm23-H1 is a candidate metastasis suppressor gene first characterized in breast cancer [18]. Nm23 protein expression was found to positively affect survival along with absence of axillary lymph node metastases and hormonal therapy in breast cancer patients. In epithelial ovarian cancers expression of $\mathrm{Nm} 23$ kinase is strongly upregulated, with $88 \%$ of ovarian cancers staining positively [18]. There is a trend towards decreased survival with focal staining of nm23 kinase. Although no statistical significance was found with nm23 protein expression in ovarian cancers, pattern and intensity of protein staining may identify patients at high risk for tumor progression [18]. 


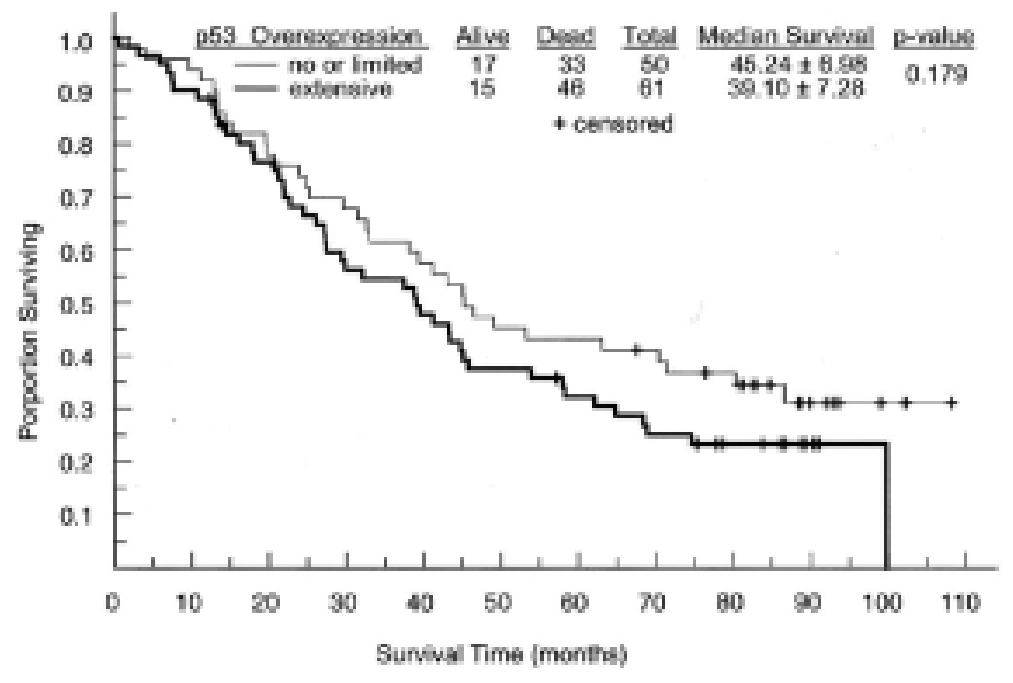

Fig. 1. Kaplan-Meier survival analysis for p53 overexpression.

\section{Receptor tyrosine kinases}

Protein kinases are enzymes that covalently attach phosphates to the side chain of serine, threonine, or tyrosine residues of specific proteins inside cells. Such phosphorylation of proteins can control their enzymatic activity, interactions with other proteins and molecules, and propensity for degradation by proteases. Perturbation of protein kinase signaling by mutations and other genetic alterations can result in deregulated kinase activity and malignant transformation.

\subsection{Receptor tyrosine kinases}

All Receptor tyrosine kinases (RTKs) share several structural features. They are glycoproteins possessing three similar components: an extracellular ligandbinding domain, which conveys ligand specificity, a single hydrophobic transmembrane domain, which anchors the receptor to the membrane, and an intracellular tyrosine kinase domain which initiates cellular signaling [19]. The intracellular sequences typically contain regulatory regions in addition to the catalytic domain. Ligand binding induces activation of the intracellular tyrosine kinase domain leading to the initiation of signaling events specific for the receptor. The RTKs have been organized into families based on sequence homology, structural characteristics, and distinct motifs in the extracellular domain. There are currently 19 known families in vertebrates. The various subfamilies include receptors for EGF, PDGF, VEGF, fibroblast growth factor and hepatocyte growth factor.
Ligand binding to a RTK induces receptor dimerization with conformational changes that result in intermolecular phosphorylation of tyrosine residues at multiple sites. Receptor heterodimerization can also occur, as reported with transforming growth factor alpha interaction with receptor heterodimers comprising HER-2 and EGFR. In malignant tumors, a number of these receptors are overexpressed or mutated, leading to abnormal cell proliferation [20].

\subsection{The epidermal growth factor receptor family (Erb family)}

One of the best characterized pathways initiating malignant change in cell is the epidermal growth factor receptor (EGFR) family of membrane proteins [21]. The EGFR family consists of four structurally similar receptor tyrosine kinase (RTK) proteins including ErbB1 (EGFR), ErbB-2 (HER2/neu), ErbB-3, and ErbB4 [21]. These receptors are activated by binding of ligands including EGF, transforming growth factor alpha, amphiregulin, and the neuregulins. Upon binding to one of these ligands, the receptors form homodimers or heterodimers, e.g. EGFR: HER2 or HER4: HER2, at the cell surface. These heterodimers may form even when only one member of the pair binds its ligand. This dimerization is thought to initiate the tyrosine kinase activation through autophosphorylation of serine residues and is accompanied by recruitment of downstream signal transduction molecules including src2, GRB2, and SH3 [21]. This complex then induces downstream signaling including activation of Ras, Raf, 
MAP kinase and ultimately gene activation [22]. The biologic effects mediated by the EGF family of receptors are quite broad, and include mediation of cell proliferation, development, differentiation, and oncogenesis [21].

\section{3. $E G F R$}

EGFR protein expression in 96 cases of ovarian cancer was present in $39.8 \%$ of cancers analyzed [23]. The clinic significance of EGFR protein expression in the development and progression of human ovarian carcinoma was studied in 7 ovarian cystadenomas, $6 \mathrm{mu}-$ cinous tumors of low malignant potential (LMP), and 25 invasive adenocarcinomas. EGF, and EGFR expression was found to be significantly higher in mucinous cystadenocarcinomas than in mucinous cystadenomas or mucinous tumors of LMP [24]. In a series of 226 patients with early stage epithelial ovarian carcinomas, FIGO stages IA-IIC, a number of clinicopathological factors were studied in relation to p53 and epidermal growth factor receptor (EGFR) protein expression [25]. In a Cox multivariate analysis, tumor grade, p53 status, and EGFR status were all independent and significant prognostic factors with regard to DFS [25].

\subsection{Her-2}

Studies have shown that the HER-2 oncogene is overexpressed in approximately $25-30 \%$ of ovarian carcinoma (OC) cases, but, to date no consensus regarding overexpression and prognosis has been possible [26, 27]. HER-2 immunohistochemical staining of ovarian tissue is primarily a cytoplasmic stain, however, there is varying intensity of the staining, requiring interpretation [26]. A recent immunohistochemical evaluation of Her-2 protein expression performed on the first 181 patients included in the Danish MALOVA study diagnosed with epithelial OC provided contrasting results [26]. HER-2 overexpression was found in $52.5 \%$ of these cases in which $74.7 \%$ were weakly positive $(1+)$ and $25.3 \%$ were moderately $(2+)$ to intensely positive $(3+)$. In this study increased HER2 expression was found to be correlated with reduced survival [26]. Significant differences in survival between patients with positive Her-2 expression and those without HER-2 overexpression were found for the subgroups of FIGO Stage I, Stage III, and Stage IV. For stage I patients HER-2 negative five-year survival approached $100 \%$ compared to $71 \%$ for HER 2 positive patients. For FIGO stage III and IV ovarian cancer patients HER-2 negative patients five year survival was approximately $45 \%$, while HER-2 positive patients survival was only $18 \%$ [26]. Multivariate survival analyses demonstrated that HER-2 overexpression to be a prognostic marker.

In another study which could explain the conflicting results regarding Her-2 protein expression and survival in the literature, Her-2 protein expression, as well as the frequency of Her-2 amplification were examined in a series of 103 high-grade, advanced-stage (FIGO stage III or IV) ovarian surface epithelial carcinomas [27]. Barely 5 of 102 (4.9\%) tumors were positive for Her-2 protein expression by immunohistochemistry. Over a third, 33.3\% tumors, however, showed Her-2 amplification. Only $25 \%$ of cancers that showed Her-2 amplification by FISH were positive for Her-2 protein overexpression by immunohistochemistry. There was no correlation between Her-2 expression and survival [27]. Thus, the strong correlation between Her-2 immunostaining and amplification characteristic of breast carcinoma might not be observed in ovarian carcinoma.

\subsection{Endothelial growth factors; vascular endothelial growth factor}

Angiogenesis is the formation of new blood vessels and this process is required by many biologic processes including the development of cancer [28]. New vessel formation can be stimulated by a variety of factors including vascular endothelial growth factor (VEGF) [29, 30]. The VEGF family of glycoproteins consists of six related growth factors, VEGF-A (known as VEFG) through VEGF-E, and placental growth factor (PIGF)1 and 2 [31]. VEGF mediates angiogenic signals to the vascular endothelium through high affinity RTKs that are thought to activate the MAPK pathway. Although many stimulators and inhibitors of angiogenesis have been identified, the trigger that causes a dormant tumor to transform into a proangiogenic tumor remains elusive [32].

Expression of vascular endothelial growth factor (VEGF) in ovarian carcinomas revealed focal or diffuse strong immunostaining in $48 \%-51 \%$ of carcinomas $[33,34]$. In early stage ovarian cancer, increased VEGF protein expression by immunohistochemistry was associated with a decreased disease free survival 18 months versus greater than 120 months for non VEGF expressors [35]. In a multivariate analysis, only VEGF expression was associated with poorer survival in these early stage ovarian cancer patients. Significant associations between the VEGF expression and disease FI- 
GO stage, histologic grade, and patient outcome has been observed [34]. The survival of patients with high VEGF expression was significantly worse than that of patients with low and negative VEGF expression. Multivariate analysis revealed that disease stage and VEGF expression were significant and independent prognostic indicators of overall survival time.

\section{Cell cycle regulators and inhibitors}

\subsection{Cyclins}

Phosphorylation of $\mathrm{Rb}$ protein by serine/threonine kinases known as cyclin-dependent kinases (cdks) inactivates $\mathrm{Rb}$. The cdks form complexes with proteins called cyclins. There are at least nine cdks (cdk1-cdk9) and 15 cyclins (cyclin A through cyclin T) [36]. Cdk4 and cdk6 along with their D-type cyclins are responsible for the cells progression through G1 phase. Cdk2 and cyclin E complex are responsible for the progression from $\mathrm{G} 1$ to $\mathrm{S}$ phase. Cdk2 and cyclin A are responsible for the cells progression through $\mathrm{S}$ phase and cdk1 and cyclin $\mathrm{B}$ are required for mitosis. These complexes are in turn inhibited by a combination of small proteins called cdk inhibitors (CKIs). The INK4 (inhibitor of cdk4) family consists of $\mathrm{p} 16^{\text {ink } 4 \mathrm{a}}, \mathrm{p} 15^{\mathrm{ink} 4 \mathrm{~b}}, \mathrm{p} 18^{\text {ink } 4 \mathrm{c}}$, and $\mathrm{p} 19^{\mathrm{ink} 4 \mathrm{~d}}$ and specifically inhibit cyclin D associated kinases. The protein kinase inhibitor protein family of p2 $1^{\text {waf1 }}$, p $27^{\mathrm{kip} 1}$, and $\mathrm{p} 57^{\mathrm{kip} 2}$ inhibit the cyclin E/cdk2 and cyclin A/cdk2 complexes. Loss of expression of CKIs confers a poor prognosis in a variety of cancers [36].

\subsection{Cyclin D}

Expression of cyclin D1 was detected in $32.4 \%$ of epithelial cancers, $69.6 \%$ borderline tumors, and $72.7 \%$ of benign tumors. Cyclin D1 expression inversely correlated with tumor grade [37]. Cyclin D1 overexpression was more frequently detected in borderline and grade 1 tumors than in grade 2 and grade 3 tumors. The expression of cyclin D1 was also examined in a consecutive series of 134 serous EOC nineteen percent of EOC were found to overexpress cyclin D1 [38]. On multivariate analysis, overexpression of cyclin D1 combined with other molecular markers to include combined loss of p21(Waf1/Cip1) in the presence of p53 overexpression were independent predictors of reduced overall survival [38]. In another study of 70 patients followed for 8 years with EOC, the cyclin D1 protein

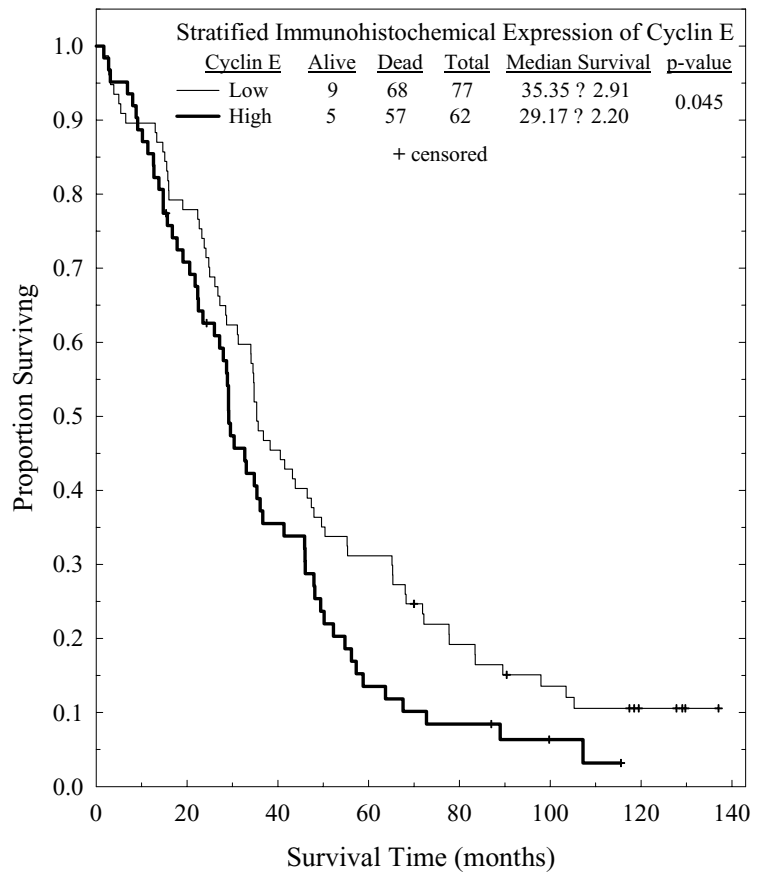

Fig. 2. Kaplan-Meier survival analysis for cyclin E expression positive tumor cells in women with suboptimally debulked advanced ovarian cancer.

content was analyzed by Western blotting [39]. Patients with highly positive cyclin D1 tumors had shorter overall survival than patients with positive cyclin D1 (median survival 31 vs. 49 month). For patients with high cyclin D1 expression and residual disease greater than $2 \mathrm{~cm}$, the relative risks of death were to 2.48 and 3.7 , respectively [39].

\subsection{Cyclin E}

Immunohistochemical expression of cyclin $\mathrm{E}$ was evaluated in 139 advanced, suboptimally debulked epithelial ovarian cancer specimens from patients treated on Gynecologic Oncology Group protocol 111 [40]. High cyclin E protein expression $(>$ or $=40 \%$ cyclin E positive tumor cells) was seen in 62 (45\%) of the advanced, suboptimally debulked ovarian cancer patients. Expression of cyclin E was not associated with age, race, stage, grade, cell type, or amount of residual disease. High verses low cyclin E expression was associated with a shorter median survival (29 versus 35 months) and worse overall survival $(P<0.05)$ (Fig. 2) [40]. High cyclin E protein expression was also associated with a decreased survival when patients stratified by FIGO stage III, Serous histology, and platinum based chemotherapy. High cyclin E expression was an in- 


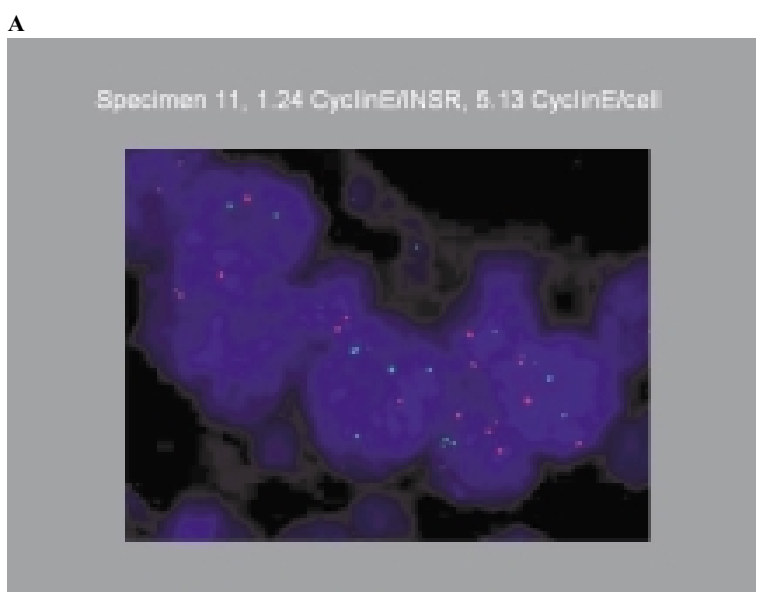

B

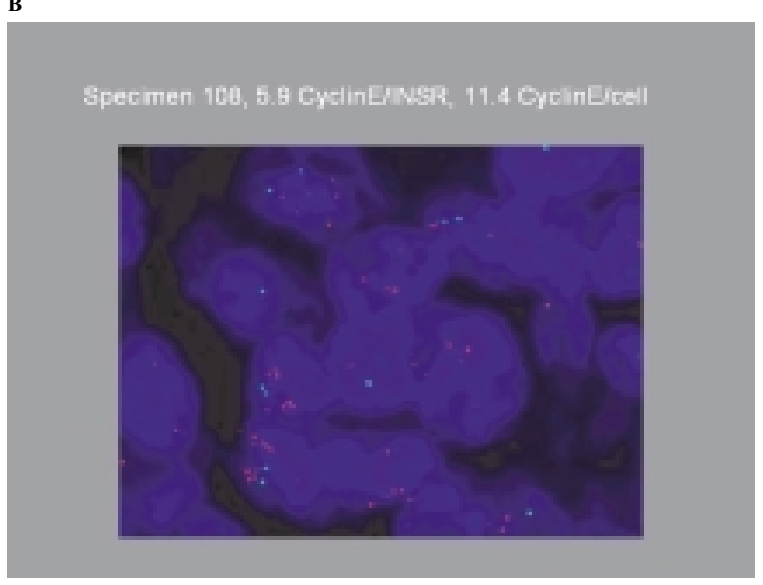

Fig. 3. FISH analysis. Representative FISH analysis of low cyclin E protein expressor by IHC, and high cyclin E protein expressors by IHC and corresponding amplification of $19 q$.

dependent poor prognostic factor for patients with advanced ovarian cancer, and it was associated with amplification of the cyclin E gene (Fig. 3) [40]. Clear cell carcinoma also exhibits significantly increased expression of cyclin E [41]. The incidence of cyclin E staining was significantly higher in clear cell carcinoma $(100 \%)$ than in either endometrioid $50 \%$, or poorly differentiated carcinoma $20 \%$.

\subsection{CKIs}

\subsection{1. $P 16$}

Approximately $40 \%-43 \%$ of epithelial ovarian cancers will overexpress p16 protein, while most benign tumors will show no p16 expression in the tumor cells [42-44]. The prognostic significance of the G1 pathway was evaluated in 59 epithelial ovarian cancer patients undergoing surgery and platinum-based chemotherapy [43]. Abnormal expression of p16 was observed in $33.9 \%$ of studied cases. Abnormal G1 pathway, alteration in $\mathrm{p} 16, \mathrm{Rb}$, or cyclinD1/cdk4 protein, was detected in $49.2 \%$ of cases. Although individually p16 protein overexpression was not of prognostic significance, univariate analyses identified abnormal G1 pathway (HR, 2.935; $P=0.03$ ) as prognostic factors for overall survival [43].

\subsection{2. $P 21, P 27$}

The CKI family of protein inhibitors p21, p27, and p57 inhibit the cdk 2 cyclin complexes. The frequency of expression and the possible prognostic significance of p21, and p27 proteins were examined in a series of 185 uniformly treated patients with stage III ovarian cancer [45]. p21 has been found to be overexpressed in $48 \%$ of cases $[45,46]$. Neither p21 nor p27 protein expression was of prognostic significance for the whole group of patients. Western blot analysis, however, of p21 protein appeared to confirm the significance of this CKI in ovarian cancer prognosis. In a series of 102 ovarian tissue samples including normal ovary, primary ovarian tumors, omental metastasis, recurrent disease and residual tumor after chemotherapy exposure p21 protein was detectable in $74 \%$ ovarian tissue samples. In the sub group of stage III-IV ovarian cancer patients, p21-positive cases showed a more favorable prognosis with the 3-year time to progression (TTP) rate was $58 \%$ for p21-positive compared with $33 \%$ of p21-negative [47].

p27Kip1 is a member of the Cip1/Kip1 family of cyclin-dependent kinase inhibitors and is a potential tumor suppressor gene. p27 protein expression has been evaluated by immunohistochemistry and Western blot analysis in a series of 82 epithelial ovarian tumors, 16 classified as low malignant potential (LMP) and 66 classified as primary ovarian adenocarcinomas [48]. Analysis revealed frequent loss of p27 expression in $33 \%$ of primary ovarian adenocarcinomas compared to only $6 \%$ of LMP tumors. In addition to nuclear staining, cytoplasmic localization of p27 was noted in 55\%. There was a significant correlation between presence of p27 staining and a longer time to progression [48]. p27Kip1 expression was detected in $47 \%$ patients with stages III-IV ovarian carcinoma [49]. Although, p27 expression did not correlate with any of the classical clinicopathological parameters, the 5-year TTP rate in p27-positive patients was $50 \%$ versus $11 \%$ in p27negative patients. p27-positive also cases showed a 5 -year OS rate of $53 \%$ compared with $43 \%$ of p27negative cases. In multivariate analysis, p27 expression 
A
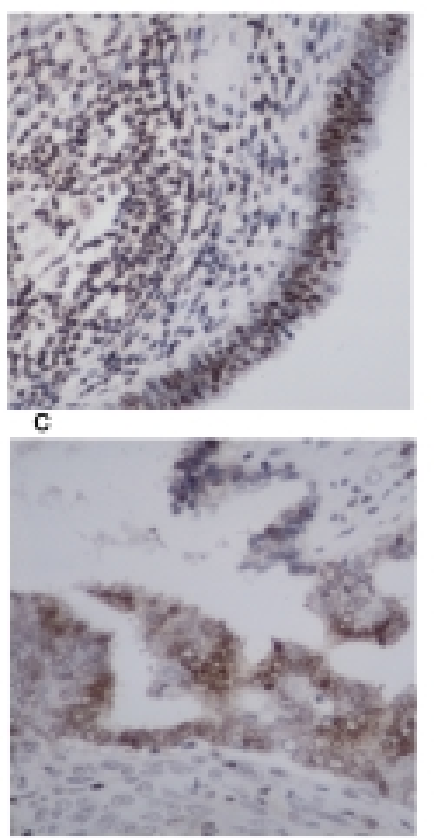

E

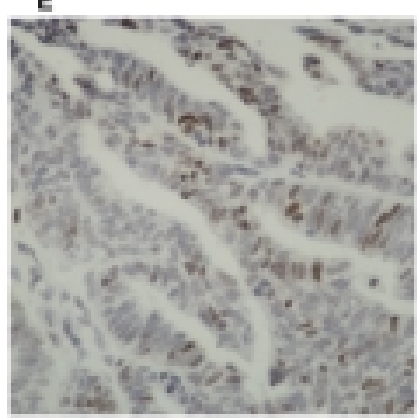

B

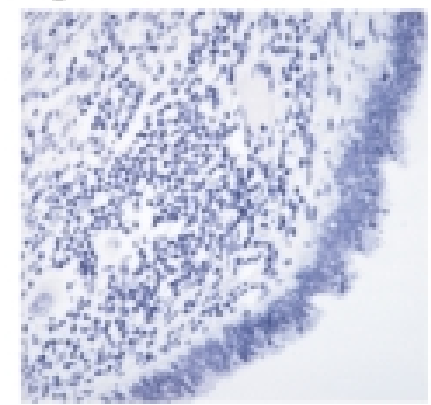

D

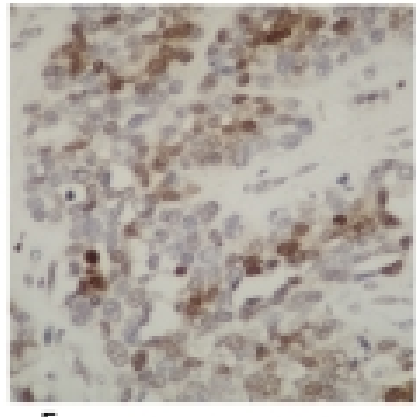

F

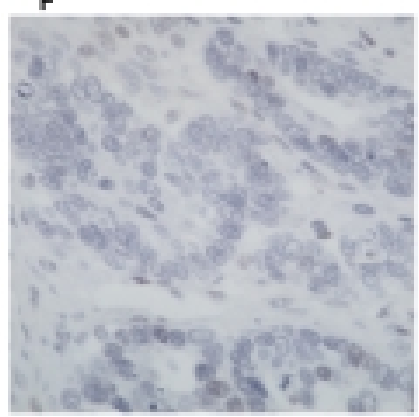

Fig. 4. Immunohistochemical expression of p27 in a benign and invasive ovarian cancers. Photomicrograph of representative p27 staining in benign ovarian epithelial tumors (A,600X), corresponding benign negative control (B,600X), epithelial low malignant potential tumors (C,700X), stage I epithelial ovarian cancer (D,1000X), stage II epithelial ovarian cancer (E,700X), and stage III epithelial adenocarcinoma (F,700X).

was an independent predictor of progression of disease and survival [49].

The location of p27 protein staining in the cell appears to be of clinical importance when interpreting p27 immunohistochemical staining (Fig. 4) [50,51]. Decreased nuclear staining has been associated with shorter survivals, as has cytoplasmic localization of p27 protein $[50,51]$. Subcellular localization of the $\mathrm{p} 27$ protein was evaluated by using tissue microarrays containing 421 cases of ovarian carcinoma [51]. Nuclear only staining was associated with a $58 \%$ five year survival, while negative ( $<5 \%$ staining) and cytoplasmic staining was associated with only a 30-32\% five year survival, (Fig. 5). The presence of p27 in the cytoplasm regardless of the nuclear stain correlated strongly with late-stage disease, extent of cytoreduction, and shorter disease-specific survival.

\section{Mitogen-Activated Protein Kinase (MAPK) Pathway, RAS Proteins}

The ras superfamily of genes encodes small GTP binding proteins that are responsible for regulation of many cellular processes, including differentiation, cytoskeletal organization, and protein trafficking. Each ras protein consists of approximately 190 amino acid residues [52]. Activated ras activates RAF, which is a serine/threonine kinase. RAF activates mitogenactivated protein kinase kinase (MAPKK), also called 


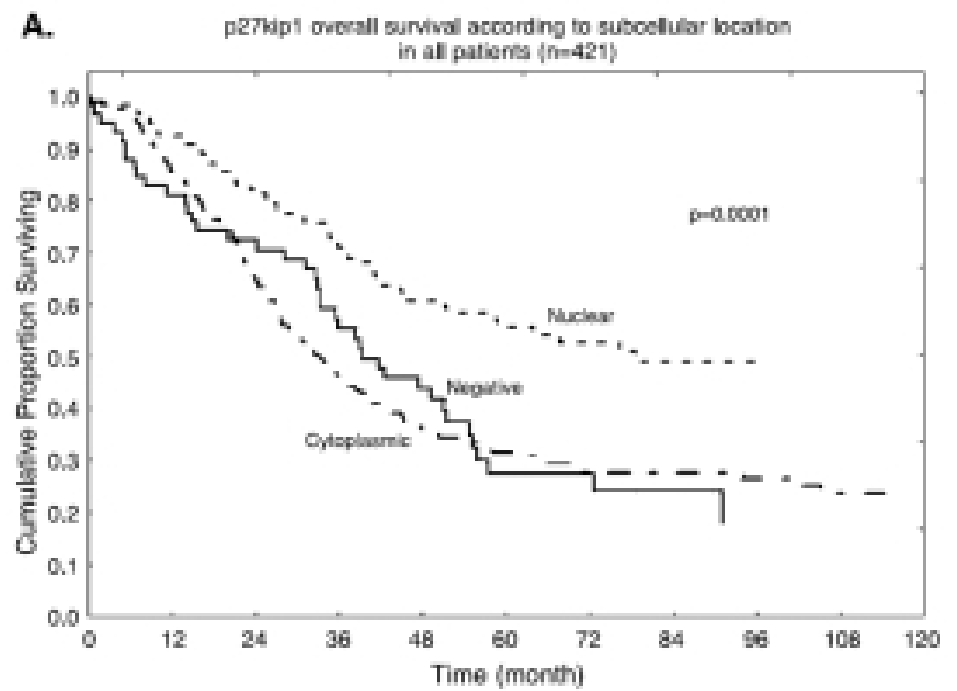

Fig. 5. Association of p27kip1 subcellular localization on overall survival in ovarian carcinoma, localization in all ovarian cancer cases.

MEK, which in turn activates mitogen activated protein (MAP) kinase or extracellular regulated kinase (ERK). The MAPK kinase (MEK), a central kinase in this pathway, is a critical signaling protein for multiple oncogenic pathways including the EGFR family, VEGF, PDGF, and activated Ras. MAP kinase activation also results in phosphorylation and activation of ribosomal S6 kinase and transcription factors such as c-JUN, $\mathrm{c}-M Y C$ and c-FOS, resulting in the switching on of a number of genes associated with proliferation. Mutant oncogenic forms of Ras ( $\mathrm{H}$-ras, $\mathrm{N}$-ras, $\mathrm{K}_{A}$-ras, $\mathrm{K}_{B}$-ras) have been found in up to $30 \%$ of all human cancers and $4 \%$ of epithelial ovarian cancers. The presence of Ras mutations in gynecologic malignancies appears to be a rare event. In one extensive study, only 1 cystadenoma (5\%), 6 LMP tumors (30\%), and 1 ovarian carcinoma (4\%) demonstrated an activated Ki-ras gene [53].

\subsection{ARHI}

No more than $20 \%$ of invasive cancers exhibit Ras mutations. Functional activation of the RAS pathway in the absence of genetic mutations has, however, been reported in a majority of ovarian cancer cell lines [54,55]. As a result, certain members of the RAS-superfamily may act as tumor suppressor genes rather than as protooncogenes. RAS homolog gene family, member I ARHI is a maternally imprinted putative human tumor suppressor gene that maps to chromosome $1 \mathrm{p} 31$ and that encodes a $26-\mathrm{kDa}$ small G protein with $60 \%$ homology to rap and ras [55]. ARHI is expressed in nor- mal ovarian epithelial cells but down regulated in most ovarian cancer cell lines [55]. ARHI protein expression is reduced in LMP tumors of the ovary, and in frankly malignant invasive ovarian cancers, ARHI protein was down-regulated in $63 \%$, and expression of the gene was lost altogether in 47\% [55]. Five-year survival of 39\% was observed in patients whose tumors had strong expression of ARHI, $43 \%$ in those with weak expression, compared with $33 \%$ in those with negative expression. ARHI expression correlated with p21 protein expression and was associated with a prolonged disease free survival but not overall survival.

\section{2. $M A P K$}

Mitogen-activated protein kinase (MAPK) plays a pivotal role in signal transduction. Forty five percent of ovarian serous carcinomas were positive for MAPK protein expression [56]. There was a lower frequency of expression of active MAPK in high-grade ovarian serous carcinomas, $41 \%$, as compared with low-grade serous tumors, $81 \%$ active MAPK expression. Active MAPK was present in all of the $19 \%$ low-grade tumors with either KRAS or BRAF mutations as well as in $41 \%$ of tumors with wild-type KRAS and BRAF [56]. In advanced FIGO stage serous ovarian carcinomas, expression of active MAPK alone served as a good survival indicator in the 2-year follow-up but not in the 5-year follow-up. Active MAPK appears to be more frequently expressed in low-grade than in high-grade ovarian serous carcinoma, and as a result may provide a 
therapeutic target in these tumors. Active MAPK could also serve as a good prognostic marker in patients with high-grade serous carcinomas.

\section{Conclusion}

With the discovery success of Herceptin in the treatment of breast cancer and Gleevec in the treatment of GIST tumors, molecular approaches to the treatment of cancer are becoming even more pervasive. Logical targeted approaches to the treatment of gynecologic malignancies are becoming the standard of care. The shotgun approach of isolated chemotherapy or radiation therapy is rapidly becoming a modality of the past. Recently, Bevacizumab, a recombinant humanized version of a murine anti-human VEGF monoclonal antibody, named rhuMAb VEGF, has displayed activity in refractory ovarian cancer [57]. This molecular agent is quickly being introduced into phase III cooperative group trials to confirm its efficacy. A minimal understanding of the basic concepts of life and death in the cell is essential for the gynecologic oncologist as we approach this new molecular era. Hopefully this review will provide some of these building blocks.

\section{References}

[1] A. Jemal, R.C. Tiwari, T. Murray, A. Ghafoor, A. Samuels, E. Ward et al., Cancer statistics, 2004, CA Cancer J Clin 54 (2004), 8-29. Notes: CORPORATE NAME: American Cancer Society.

[2] W.P. McGuire, W.J. Hoskins, M.F. Brady, P.R. Kucera, E.E. Partridge, K.Y. Look et al., Comparison of combination therapy with paclitaxel and cisplatin versus cyclophosphamide and cisplatin in patients with suboptimal stage III and stage IV ovarian cancer: a Gynecologic Oncology Group study, Semin Oncol 24 (1997), S2-13-S2-16.

[3] M.A. Bookman, Biologic therapies for gynecologic cancer, Curr Opin Oncol 7 (1995), 478-484.

[4] M.A. Bookman, Biological therapy for gynecologic malignancies, Cancer Treat Res 95 (1998), 115-147.

[5] A. Berchuck, M.F. Kohler, J.R. Marks, R. Wiseman, J. Boyd and R.C. Bast, Jr., The p53 tumor suppressor gene frequently is altered in gynecologic cancers, Am J Obstet Gynecol 170 (1994), 246-252.

[6] G. Casey, M.E. Lopez, J.C. Ramos, S.J. Plummer, M.J. Arboleda, M. Shaughnessy et al., DNA sequence analysis of exons 2 through 11 and immunohistochemical staining are required to detect all known p53 alterations in human malignancies, Oncogene 13 (1996), 1971-1981.

[7] G.H. Eltabbakh, J.L. Belinson, A.W. Kennedy, C.V. Biscotti, G. Casey, R.R. Tubbs et al., p53 overexpression is not an independent prognostic factor for patients with primary ovarian epithelial cancer, Cancer 80 (1997), 892-898.
[8] J.P. Geisler, H.E. Geisler, G.A. Miller, M.C. Wiemann, Z. Zhou and W. Crabtree, p53 and bcl-2 in epithelial ovarian carcinoma: their value as prognostic indicators at a median follow-up of 60 months, Gynecol Oncol 77 (2000), 278-282.

[9] M. Baekelandt, G.B. Kristensen, J.M. Nesland, C.G. Trope and R. Holm, Clinical significance of apoptosis-related factors p53, Mdm2, and Bcl-2 in advanced ovarian cancer, J Clin Oncol 17 (1999), 2061.

[10] L.C. Hartmann, K.C. Podratz, G.L. Keeney, N.A. Kamel, J.H. Edmonson, J.P. Grill et al., Prognostic significance of p53 immunostaining in epithelial ovarian cancer, J Clin Oncol 12 (1994), 64-69.

[11] L. Havrilesky, M. Darcy, H. Hamdan, R.L. Priore, J. Leon, J. Bell et al., Prognostic significance of p53 mutation and p53 overexpression in advanced epithelial ovarian cancer: a Gynecologic Oncology Group Study, J Clin Oncol 21 (2003), 38143825. Notes: CORPORATE NAME: Gynecologic Oncology Group Study.

[12] D. Romagnolo, L.A. Annab, T.E. Thompson, J.I. Risinger, L.A. Terry, J.C. Barrett et al., Estrogen upregulation of BRCA1 expression with no effect on localization, Mol Carcinog 22 (1998), 102-109.

[13] C. Wang, A. Horiuchi, T. Imai, S. Ohira, K. Itoh, T. Nikaido et al., Expression of BRCA1 protein in benign, borderline, and malignant epithelial ovarian neoplasms and its relationship to methylation and allelic loss of the BRCA1 gene, J Pathol 202(2) (2004), 215-223. 0022-3417.

[14] R.L. Baldwin, E. Nemeth, H. Tran, H. Shvartsman, I. Cass, $\mathrm{S}$. Narod et al., BRCA1 promoter region hypermethylation in ovarian carcinoma: a population-based study, Cancer Res $\mathbf{6 0}$ (2000), 5329-5333.

[15] G. D’Andrilli, V. Masciullo, L. Bagella, T. Tonini, C. Minimo, G.F. Zannoni et al., Frequent loss of pRb2/p130 in human ovarian carcinoma, Clin Cancer Res 10 (2004), 3098-3103.

[16] G. Peiro, J. Diebold and U. Lohrs, CAS (cellular apoptosis susceptibility) gene expression in ovarian carcinoma: Correlation with 20q13.2 copy number and cyclin D1, p53, and Rb protein expression, Am J Clin Pathol 118 (2002), 922-929.

[17] T. Tonini, C. Gabellini, L. Bagella, G. D'Andrilli, V. Masciullo, G. Romano et al., pRb2/p130 decreases sensitivity to apoptosis induced by camptothecin and doxorubicin but not by taxol, Clin Cancer Res 10 (2004), 8085-8093.

[18] P.J. Srivatsa, W.A. Cliby, G.L. Keeney, M.K. Dodson, V.J. Suman, P.C. Roche et al., Elevated nm 23 protein expression is correlated with diminished progression-free survival in patients with epithelial ovarian carcinoma, Gynecol Oncol 60 (1996), 363-372.

[19] D. Fabbro, D. Parkinson and A. Matter, Protein tyrosine kinase inhibitors: new treatment modalities? Curr Opin Pharmacol 2 (2002), 374-381.

[20] S. Goel, S. Mani and R. Perez-Soler, Tyrosine kinase inhibitors: a clinical perspective, Curr Oncol Rep 4 (2002), 9-19.

[21] C.L. Arteaga, F. Khuri, G. Krystal and S. Sebti, Overview of rationale and clinical trials with signal transduction inhibitors in lung cancer, Semin Oncol 29 (2002), 15-26.

[22] C.L. Vogel, M.A. Cobleigh, D. Tripathy, J.C. Gutheil, L.N. Harris, L. Fehrenbacher et al., Efficacy and safety of trastuzumab as a single agent in first-line treatment of HER2overexpressing metastatic breast cancer, J Clin Oncol 20 (2002), 719-726.

[23] O.J. Owens, C. Stewart, R.E. Leake and A.M. McNicol, A comparison of biochemical and immunohistochemical assess- 
ment of EGFR expression in ovarian cancer, Anticancer Res 12 (1992), 1455-1458.

[24] H. Niikura, H. Sasano, S. Sato and A. Yajima, Expression of epidermal growth factor-related proteins and epidermal growth factor receptor in common epithelial ovarian tumors, Int $J$ Gynecol Pathol 16 (1997), 60-68.

[25] I. Skirnisdottir, T. Seidal and B. Sorbe, A new prognostic model comprising p53, EGFR, and tumor grade in early stage epithelial ovarian carcinoma and avoiding the problem of inaccurate surgical staging, Int J Gynecol Cancer 14 (2004), 259-270.

[26] E.V. Hogdall, L. Christensen, S.K. Kjaer, J. Blaakaer, J.E. Bock, E. Glud et al., Distribution of HER-2 overexpression in ovarian carcinoma tissue and its prognostic value in patients with ovarian carcinoma: from the Danish MALOVA Ovarian Cancer Study, Cancer 98 (2003), 66-73.

[27] C.H. Lee, D.G. Huntsman, M.C. Cheang, R.L. Parker, L. Brown, P. Hoskins et al., Assessment of Her-1, Her-2, And Her-3 expression and Her-2 amplification in advanced stage ovarian carcinoma, Int J Gynecol Pathol 24 (2005), 147-152.

[28] P.J. Paley, Angiogenesis in ovarian cancer: molecular pathology and therapeutic strategies, Curr Oncol Rep 4 (2002), 165174.

[29] M. Pegram, S. Hsu, G. Lewis, R. Pietras, M. Beryt, M. Sliwkowski et al., Inhibitory effects of combinations of HER2/neu antibody and chemotherapeutic agents used for treatment of human breast cancers, Oncogene 18 (1999), 22412251 .

[30] Z. Aguilar, R.W. Akita, R.S. Finn, B.L. Ramos, M.D. Pegram, F.F. Kabbinavar et al., Biologic effects of heregulin/neu differentiation factor on normal and malignant human breast and ovarian epithelial cells, Oncogene 18 (1999), 6050-6062.

[31] N. Starling and D. Cunningham, Monoclonal antibodies against vascular endothelial growth factor and epidermal growth factor receptor in advanced colorectal cancers: present and future directions, Curr Opin Oncol 16 (2004), 385-390.

[32] R.J. D'Amato, M.S. Loughnan, E. Flynn and J. Folkman, Thalidomide is an inhibitor of angiogenesis, Proc Natl Acad Sci USA 91 (1994), 4082-4085.

[33] H. Brustmann, Vascular endothelial growth factor expression in serous ovarian carcinoma: relationship with topoisomerase II alpha and prognosis, Gynecol Oncol 95 (2004), 16-22.

[34] G.H. Shen, M. Ghazizadeh, O. Kawanami, H. Shimizu, E. Jin, T. Araki et al., Prognostic significance of vascular endothelial growth factor expression in human ovarian carcinoma, $\mathrm{Br} \mathrm{J}$ Cancer 83 (2000), 196-203.

[35] P.J. Paley, K.A. Staskus, K. Gebhard, D. Mohanraj, L.B. Twiggs, L.F. Carson et al., Vascular endothelial growth factor expression in early stage ovarian carcinoma, Cancer 80 (1997), 98-106.

[36] J.H. Farley and M.J. Birrer, Biologic directed therapies in gynecologic oncology, Curr Oncol Rep 5 (2003), 459-467.

[37] L. Sui, M. Tokuda, M. Ohno, O. Hatase and T. Hando, The concurrent expression of p27(kip1) and cyclin D1 in epithelial ovarian tumors, Gynecol Oncol 73 (1999), 202-209.

[38] G. Ferrandina, F. Legge, E. Martinelli, F.O. Ranelletti, G.F. Zannoni, L. Lauriola et al., Survivin expression in ovarian cancer and its correlation with clinico-pathological, surgical and apoptosis-related parameters, Br J Cancer 92 (2005), 271277.

[39] F. Barbieri, P. Lorenzi, N. Ragni, G. Schettini, C. Bruzzo, F. Pedulla et al., Overexpression of cyclin D1 is associated with poor survival in epithelial ovarian cancer, Oncology 66 (2004), $310-315$.
[40] J. Farley, L.M. Smith, K.M. Darcy, E. Sobel, D. O'Connor, B. Henderson et al., Cyclin E expression is a significant predictor of survival in advanced, suboptimally debulked ovarian epithelial cancers: a Gynecologic Oncology Group study, Cancer Res 63 (2003), 1235-1241. Notes: CORPORATE NAME: Gynecologic Oncology Group.

[41] M. Shimizu, T. Nikaido, T. Toki, T. Shiozawa and S. Fujii, Clear cell carcinoma has an expression pattern of cell cycle regulatory molecules that is unique among ovarian adenocarcinomas, Cancer 85 (1999), 669-677.

[42] Y. Dong, M.D. Walsh, M.A. McGuckin, B.G. Gabrielli, M.C. Cummings, R.G. Wright et al., Increased expression of cyclin-dependent kinase inhibitor 2 (CDKN2A) gene product $\mathrm{P} 16 \mathrm{INK} 4 \mathrm{~A}$ in ovarian cancer is associated with progression and unfavourable prognosis, Int J Cancer 74 (1997), 57-63.

[43] T. Kusume, H. Tsuda, M. Kawabata, T. Inoue, N. Umesaki, T. Suzuki et al., The p16-cyclin D1/CDK4-pRb pathway and clinical outcome in epithelial ovarian cancer, Clin Cancer Res 5 (1999), 4152-4157.

[44] L. Sui, Y. Dong, M. Ohno, M. Goto, T. Inohara, K. Sugimoto et al., Inverse expression of $\mathrm{Cdk} 4$ and p16 in epithelial ovarian tumors, Gynecol Oncol 79 (2000), 230-237.

[45] M. Baekelandt, R. Holm, C.G. Trope, J.M. Nesland and G.B. Kristensen, Lack of independent prognostic significance of p21 and p27 expression in advanced ovarian cancer: an immunohistochemical study, Clin Cancer Res 5 (1999), 28482853.

[46] A. Harlozinska, J.K. Bar, M. Montenarh and S. Kartarius, Relations between immunologically different p53 forms, p21(WAF1) and PCNA expression in ovarian carcinomas, Oncol Rep 9 (2002), 1173-1179.

[47] G. Ferrandina, A. Stoler, A. Fagotti, F. Fanfani, R. Sacco, A. De Pasqua et al., p21WAF1/CIP1 protein expression in primary ovarian cancer, Int J Oncol 17 (2000), 1231-1235.

[48] V. Masciullo, A. Sgambato, C. Pacilio, B. Pucci, G. Ferrandina, J. Palazzo et al., Frequent loss of expression of the cyclindependent kinase inhibitor p27 in epithelial ovarian cancer, Cancer Res 59 (1999), 3790-3794.

[49] V. Masciullo, G. Ferrandina, B. Pucci, F. Fanfani, S. Lovergine, J. Palazzo et al., p27Kip1 expression is associated with clinical outcome in advanced epithelial ovarian cancer: multivariate analysis, Clin Cancer Res 6 (2000), 4816-4822.

[50] J.A. Hurteau, B.M. Allison, S.A. Brutkiewicz, M.G. Goebl, D.K. Heilman, R.M. Bigsby et al., Expression and subcellular localization of the cyclin-dependent kinase inhibitor p27(Kip1) in epithelial ovarian cancer, Gynecol Oncol 83 (2001), 292-298.

[51] D.G. Rosen, G. Yang, K.Q. Cai, R.C. Bast, Jr., D.M. Gershenson, E.G. Silva et al., Subcellular localization of p27kip1 expression predicts poor prognosis in human ovarian cancer, Clin Cancer Res 11 (2005), 632-637.

[52] A.A. Adjei and M. Hidalgo, Intracellular signal transduction pathway proteins as targets for cancer therapy, J Clin Oncol 23 (2005), 5386-5403.

[53] M.G. Teneriello, M. Ebina, R.I. Linnoila, M. Henry, J.D. Nash, R.C. Park et al., p53 and Ki-ras gene mutations in epithelial ovarian neoplasms, Cancer Res 53 (1993), 3103-3108.

[54] I.N. Mammas, A. Zafiropoulos and D.A. Spandidos, Involvement of the ras genes in female genital tract cancer, Int J Oncol 26 (2005), 1241-1255.

[55] D.G. Rosen, L. Wang, A.N. Jain, K.H. Lu, R.Z. Luo, Y. Yu et al., Expression of the tumor suppressor gene ARHI in epithelial ovarian cancer is associated with increased expression 
of $\mathrm{p} 21 \mathrm{WAF} 1 / \mathrm{CIP} 1$ and prolonged progression-free survival, Clin Cancer Res 10 (2004), 6559-6566.

[56] C.Y. Hsu, R. Bristow, M.S. Cha, B.G. Wang, C.L. Ho, R.J. Kurman et al., Characterization of active mitogen-activated protein kinase in ovarian serous carcinomas, Clin Cancer Res 10 (2004), 6432-6436.
[57] B.J. Monk, D.C. Choi, G. Pugmire and R.A. Burger, Activity of bevacizumab (rhuMAB VEGF) in advanced refractory epithelial ovarian cancer, Gynecol Oncol 96 (2005), 902-905. 


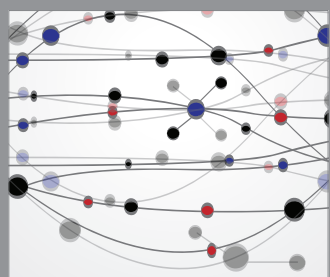

The Scientific World Journal
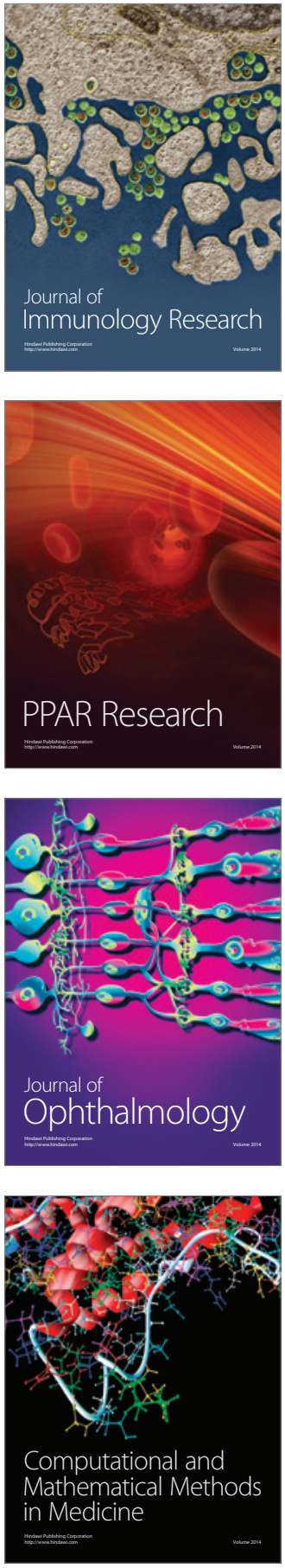

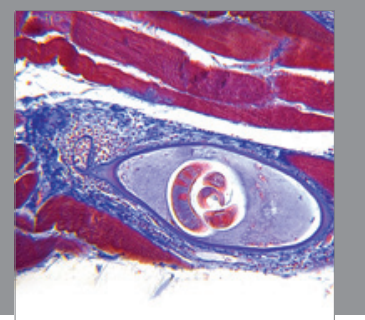

Gastroenterology

Research and Practice
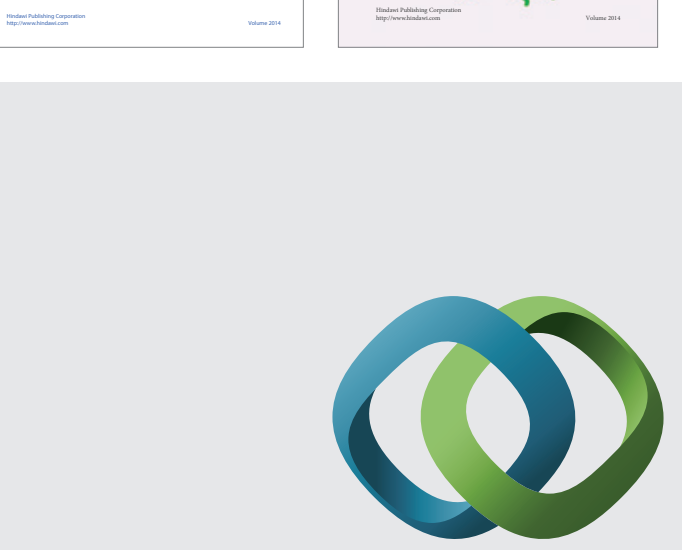

\section{Hindawi}

Submit your manuscripts at

http://www.hindawi.com
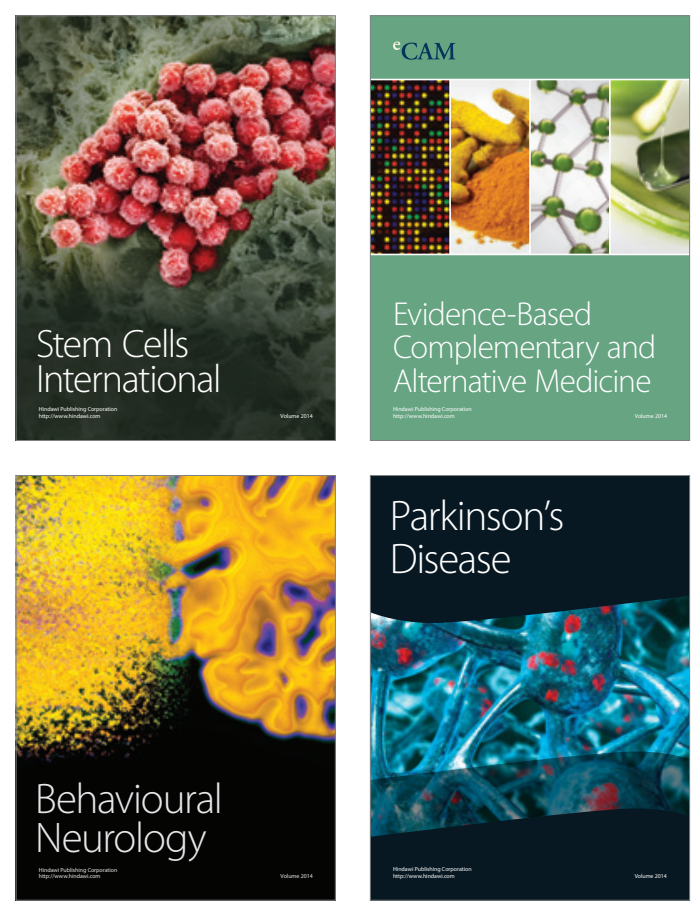

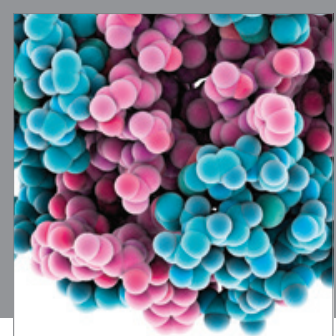

Journal of
Diabetes Research

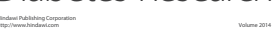

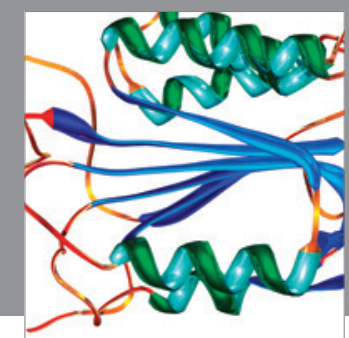

Disease Markers
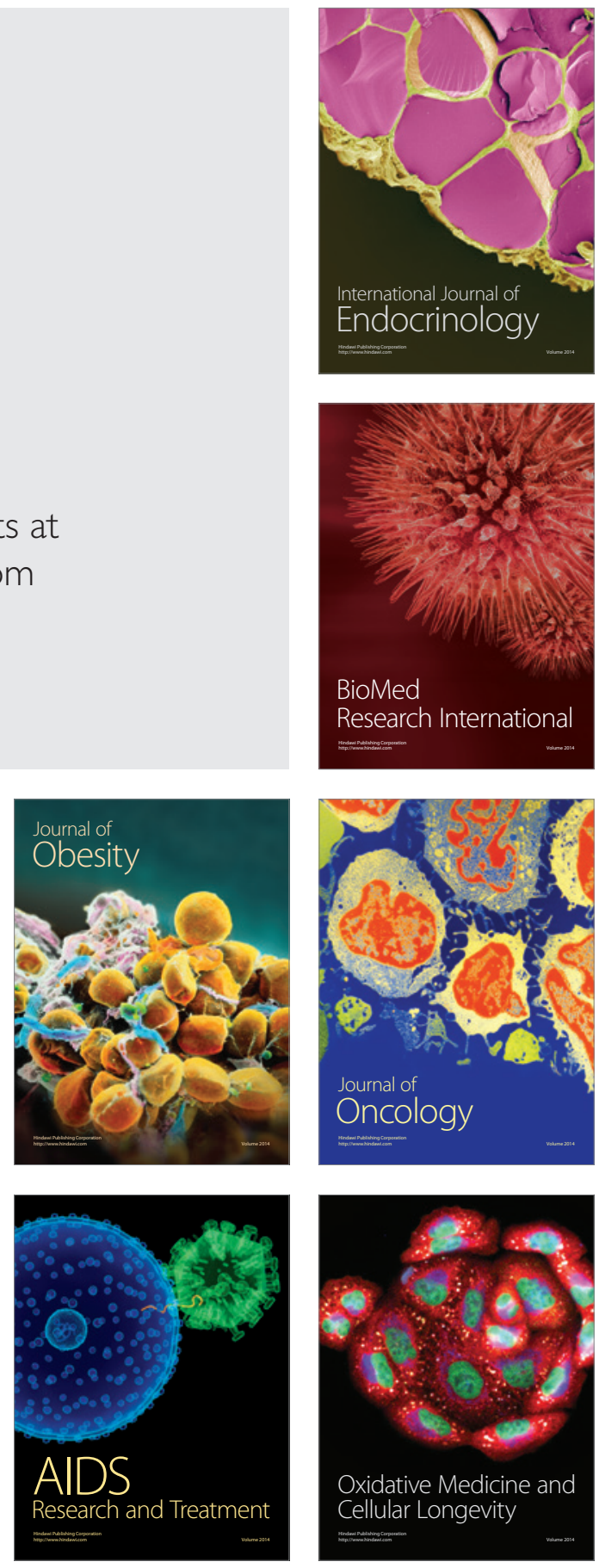